\title{
Pathology of Breast Carcinoma: Diagnostic, Prognostic, and Therapeutic Issues and Challenges
}

\author{
Rohit Bhargava, ${ }^{1}$ Sunati Sahoo, ${ }^{2}$ Nicole Nicosia Esposito, ${ }^{3}$ and Beiyun Chen ${ }^{4}$ \\ ${ }^{1}$ Department of Pathology, Magee-Womens Hospital, Pittsburgh, PA 15213, USA \\ ${ }^{2}$ Department of Pathology, Southwestern Medical Center, University of Texas, Dallas, TX 75390, USA \\ ${ }^{3}$ Department of Pathology, University of South Florida, Tampa, FL 33612, USA \\ ${ }^{4}$ Department of Pathology, Mayo Clinic, Rochester, MN 55905, USA \\ Correspondence should be addressed to Rohit Bhargava, rbhargava@mail.magee.edu
}

Received 4 April 2011; Accepted 18 June 2011

Copyright ( 92011 Rohit Bhargava et al. This is an open access article distributed under the Creative Commons Attribution License, which permits unrestricted use, distribution, and reproduction in any medium, provided the original work is properly cited.

Worldwide, breast cancer is the most common cancer affecting women. The disease is more common in the affluent world, but breast cancer incidence is steadily rising in the developing world. Breast cancer is a heterogeneous disease morphologically, immunohistochemically, and also at the molecular level regardless of the women's race or geographic location. Therefore, the prognosis and response to standard treatments can be highly variable from one patient to another. The first step towards personalized therapy for breast cancer is to understand the heterogeneity of the disease. Pathologists have been aware of breast cancer heterogeneity for a long time and, therefore, devised a grading system to identify tumors with different prognoses. Breast cancer grading is still very useful clinically (as illustrated in one of the original manuscripts in this issue by M. Purdom et al.), but additional prognostic/predictive markers have further improved our ability to personalize breast cancer therapy. The division of breast tumors into estrogen receptor (ER) positive and estrogen receptor-negative groups was an important step in recognizing heterogeneity and use of antiestrogen therapy in ER-positive disease is the first example of targeted therapy in human cancers. Recent advances in gene expression profiling have further exemplified the heterogeneity of breast cancer. The ER-positive disease is now split into at least 2 groups, one with good prognosis (likely luminal A type tumors) and another with poor prognosis (likely luminal B type tumors). The ER-negative disease is also split into an HER2-enriched category and the so-called basal-like breast cancer category. Although the molecular classes described using gene expression profiling do have morphologic and immunohistochemical correlates, there is lack of consensus on a defined morphologic or immunohistochemical criteria. Nevertheless, the molecular and subsequent immunohistochemical studies provided a framework for studying various other parameters or novel biological markers with respect to the best known prognostic/predictive markers ER, progesterone receptor (PR), and HER2. Two original articles in this issue have used similar immunohistochemical definitions to address the issue of multifocality in breast cancer ( $\mathrm{T}$. Tot et al.) and to study the prevalence of basal-like breast cancer in Sudan (K. D. Awadelkarim et al.).

The recent advances in breast cancer research has increased our understanding of breast cancer and provided us with more refined classifications. However, classifications do not immediately help breast cancer patients, but the hope is that understanding the molecular network will pave way for more targeted treatments. Therefore, studies of novel breast cancer markers are always useful. One review article and two original articles addressing these issues are included in the current issue (review by B. Uziely at al. and original articles by Y. Y. Wang et al. and by A. Nasir et al.).

In addition to the study of novel markers, traditional issues related to breast cancer are also discussed in this issue. Despite the identification of novel markers that provides insight into breast tumor biology, lymph-node metastasis still remains one of most important prognostic factor in breast cancer. V. Van Belle et al. report on the issue of ratio of positive versus negative lymph nodes by performing a comparative study of nottingham prognostic index (NPI) versus log odds prognostic index (Lpi) for short-term breast cancer-specific disease free survival. Two review articles 
(one by P. Tai et al. and another by G. Peclivanides et al.) discuss the issues related to sentinel lymph node biopsy and assessment.

Another common, but important issue in breast cancer is assessment of HER2 (ERBB2) oncogene. HER2 gene is amplified/overexpressed in approximately $20 \%$ of breast cancer and is a marker of aggressive disease. However, with the availability of HER2-targeted therapy, trastuzumab, the natural history of HER2 disease has been significantly altered. Trastuzumab treatment is highly effective in HER2 positive tumor, but it is generally ineffective in HER2negative disease. Moreover, the treatment is expensive and potentially cardiotoxic, so patient selection is very important. Due to the availability of such an effective treatment, it is very important for pathologists to be aware of all HER2-testing issues. The article on HER2 testing in breast cancer by S. Shah and B. Chen provides a comprehensive review on the subject.

Other review articles included in this issue update the reader on less well-known but increasing-talked-about topics in recent months, such as angiogenesis in breast cancer (E. S. Ch'ng et al.), circulating tumor cells in breast cancer (E. S. Graves et al.), and breast cancer metastasis to the brain (F. Arshad et al.). Treatment of breast cancer metastasis is discussed by A. M. Stark.

The scope of breast cancer research is quite broad, and, is difficult to include all topics in one issue. Nevertheless, we have tried to include both common and uncommon topics in a mix of high-quality original and review articles to satisfy the need of readers with different backgrounds. We hope the readers will find this special issue on breast cancer stimulating.

\author{
Rohit Bhargava \\ Sunati Sahoo \\ Nicole Nicosia Esposito \\ Beiyun Chen
}




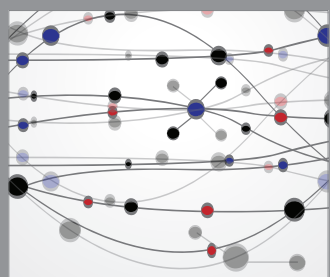

The Scientific World Journal
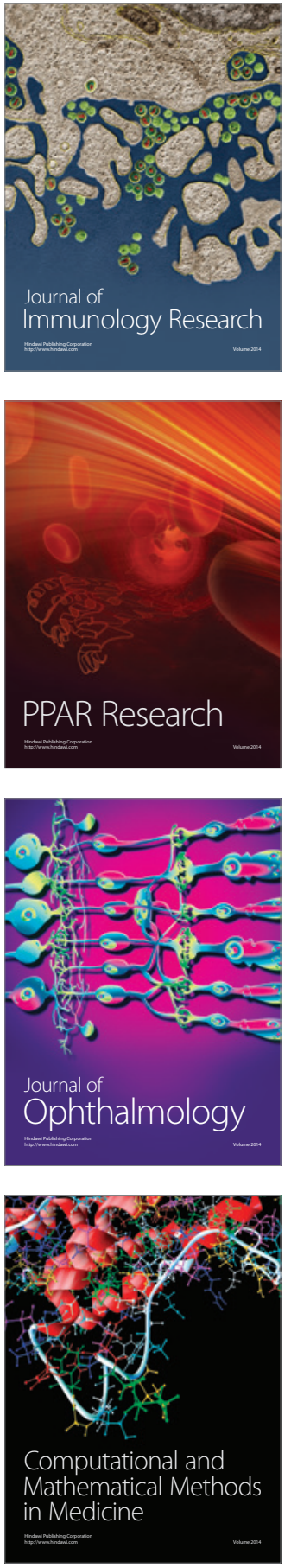

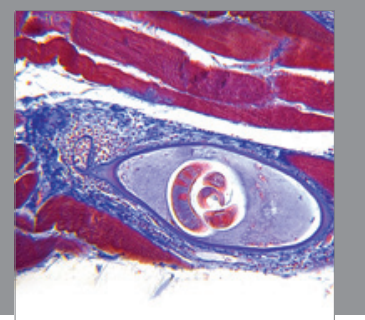

Gastroenterology

Research and Practice
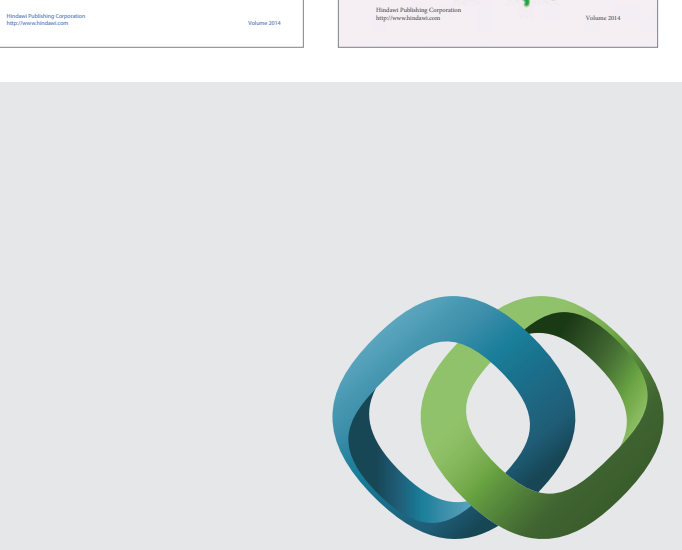

\section{Hindawi}

Submit your manuscripts at

http://www.hindawi.com
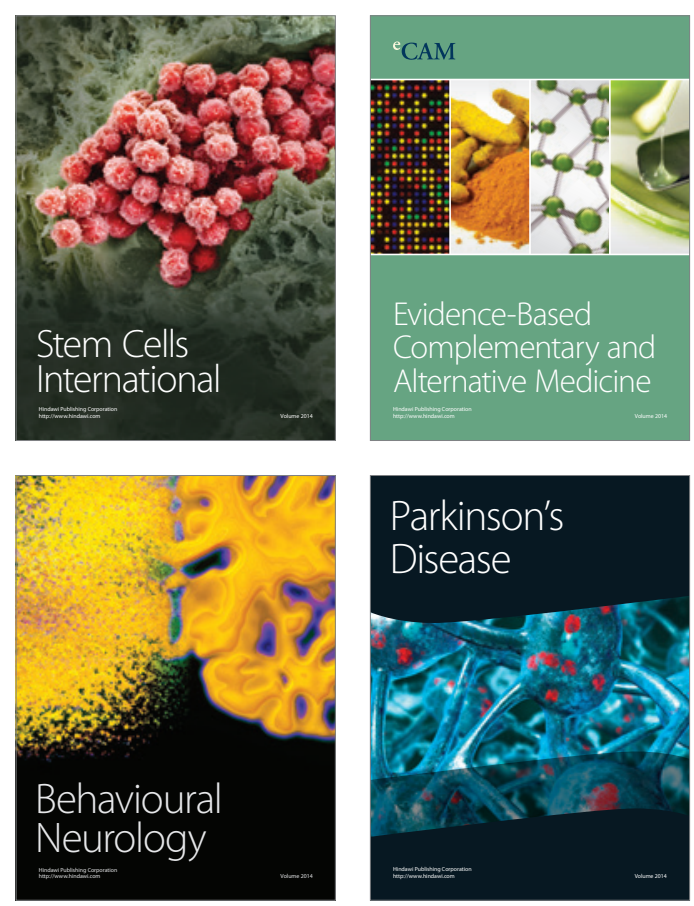

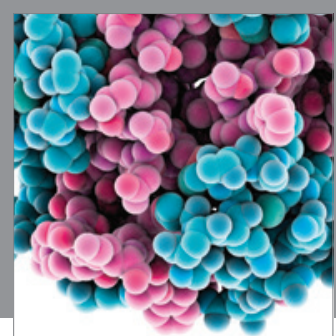

Journal of
Diabetes Research

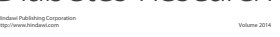

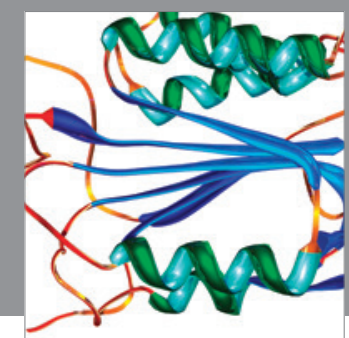

Disease Markers
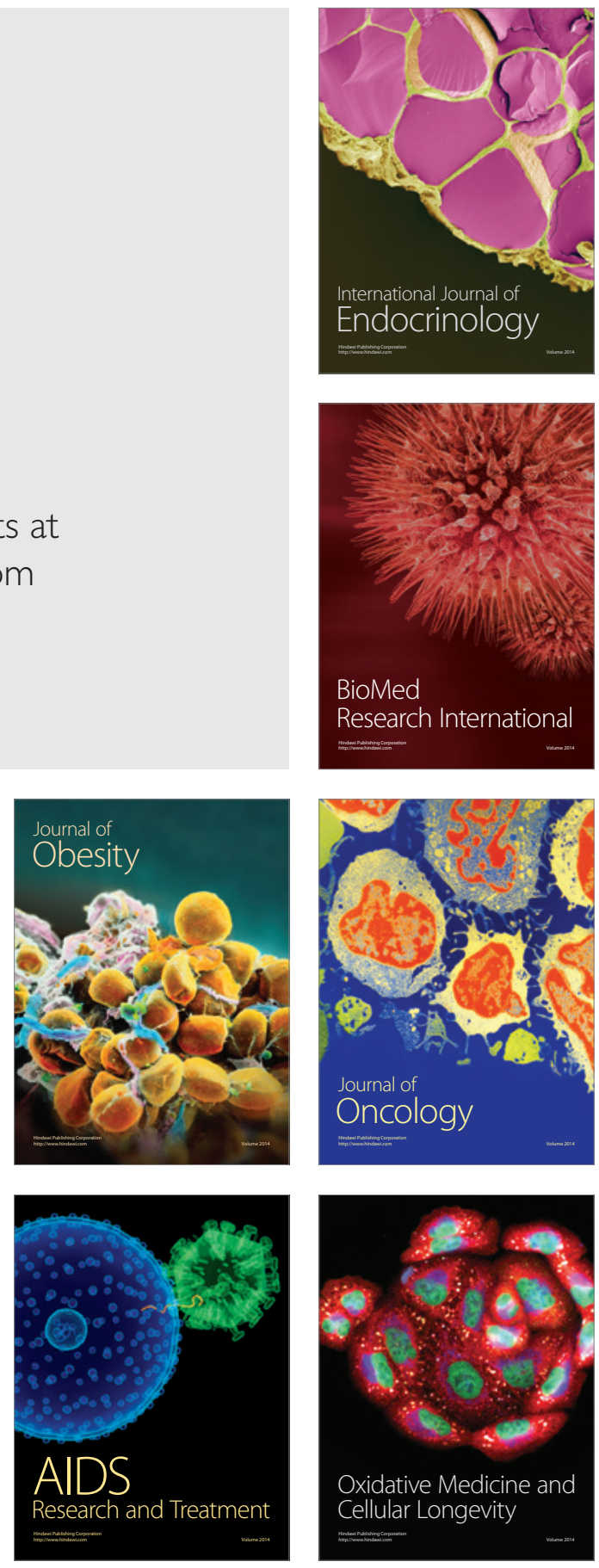